\title{
Biologic Entity Date Of Death
}

National Cancer Institute

\section{Source}

National Cancer Institute. Biologic Entity Date Of Death. NCI Thesaurus. Code C94185.

The date (and time) on which the biologic entity died. 